\title{
Neonatal repair of unilateral pulmonary vein atresia with associated coarctation of the aorta
}

\author{
Peter Chiu, MD, ${ }^{\mathrm{a}}$ Nikola Tede, MD, ${ }^{\mathrm{b}}$ and Katsuhide Maeda, MD, PhD, ${ }^{\mathrm{a}}$ Stanford, Calif
}

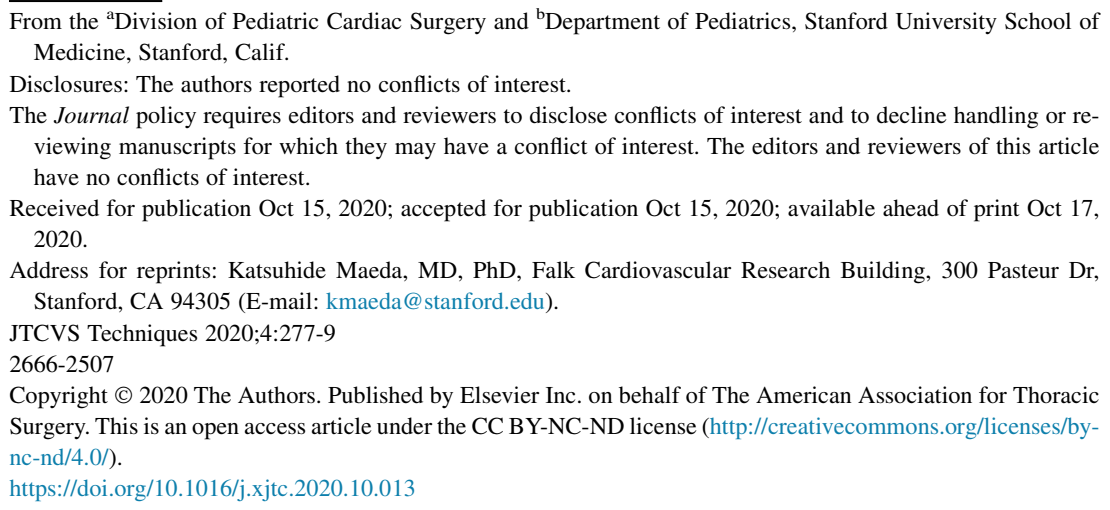

Unilateral pulmonary venous atresia is a rare entity. Patient presentation may range from recurrent pneumonia to severe respiratory compromise, but asymptomatic presentations have also been reported. ${ }^{1,2}$ The clinical course is typically dictated by age at diagnosis; patients younger than 12 to 18 months at the time of diagnosis have a particularly poor prognosis, with mortality during follow-up ranging from $65 \%$ to $76 \%{ }^{1,3}$ Associated congenital cardiac lesions may include atrial septal defect, ventricular septal defect, atrioventricular septal defect, tricuspid atresia, anomalous pulmonary veins, transposition of the great arteries, interrupted inferior vena cava, patent ductus arteriosus, bicuspid aortic valve, and Shone's complex. ${ }^{1,4}$ Here we describe the first case reported in the literature of unilateral pulmonary venous atresia with associated coarctation of the aorta undergoing neonatal repair. Informed consent for publication of the study data was obtained from the patient's guardian.

\section{CASE DESCRIPTION}

The patient was initially evaluated in utero with fetal echocardiography, which revealed coarctation of the aorta with an associated left pulmonary vein abnormality. The patient was born full term at 40 weeks and 1 day, and prostaglandin therapy was immediately initiated. The patient experienced respiratory distress requiring administration of supplemental oxygen. Chest X-ray revealed nearly complete opacification of the left lung (Figure 1,A). Echocardiography performed on day of life 1 revealed hypoplasia of the distal transverse arch ( $z$-score: -3.4$)$ extending through the isthmus. The right-sided pulmonary veins returned normally to the left atrium; the left pulmonary veins were not visualized in the normal plane. Computed tomography

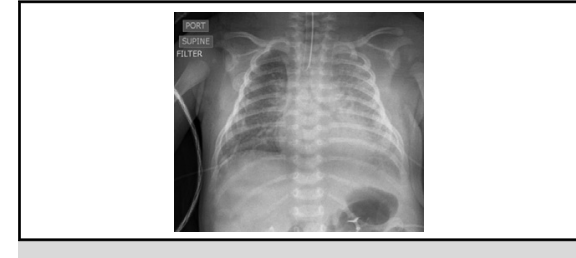

Unilateral pulmonary vein atresia in a neonate.

$$
\begin{aligned}
& \text { CENTRAL MESSAGE } \\
& \text { Neonatal pulmonary vein atresia } \\
& \text { has a poor prognosis. Pneumo- } \\
& \text { nectomy and simultaneous } \\
& \text { repair of coarctation of the aorta } \\
& \text { is a feasible approach. }
\end{aligned}
$$

See Commentary on page 280.

angiography demonstrated no evident left-sided pulmonary veins connected to the left atrium, and pulmonary angiography revealed atretic left pulmonary veins with a stenotic connection to a vertical vein connecting to the left innominate vein (Figure $1, B$ ). The mean pulmonary artery pressure was $35 \mathrm{~mm} \mathrm{Hg}$.

After a thorough discussion of the risks, benefits, and alternatives to surgical therapy with the patient's parents, the patient was brought to the operating room for repair on day of life 6. A median sternotomy was performed. After systemic heparinization, the innominate artery and the right atrium were cannulated for cardiopulmonary bypass, and the patient was cooled to $25^{\circ} \mathrm{C}$. During cooling, the left pleura was opened, and no pulmonary veins were seen (Figure 1,C). A left pneumonectomy was performed, and the specimen was noted to be very boggy and edematous (Figure 1,D). Antegrade del Nido cardioplegia was administered to achieve cold diastolic arrest, and the innominate artery was clamped for antegrade cerebral perfusion. An extended end-to-end anastomosis was performed in the usual fashion, and the patient was rewarmed and weaned from cardiopulmonary bypass without difficulty. Postoperatively, the patient recovered well and was discharged on postoperative day 32 .

Pathological review of the lung specimen revealed dilated vascular spaces located in the subpleural, interlobular, perivascular, and peribronchiolar spaces, and 


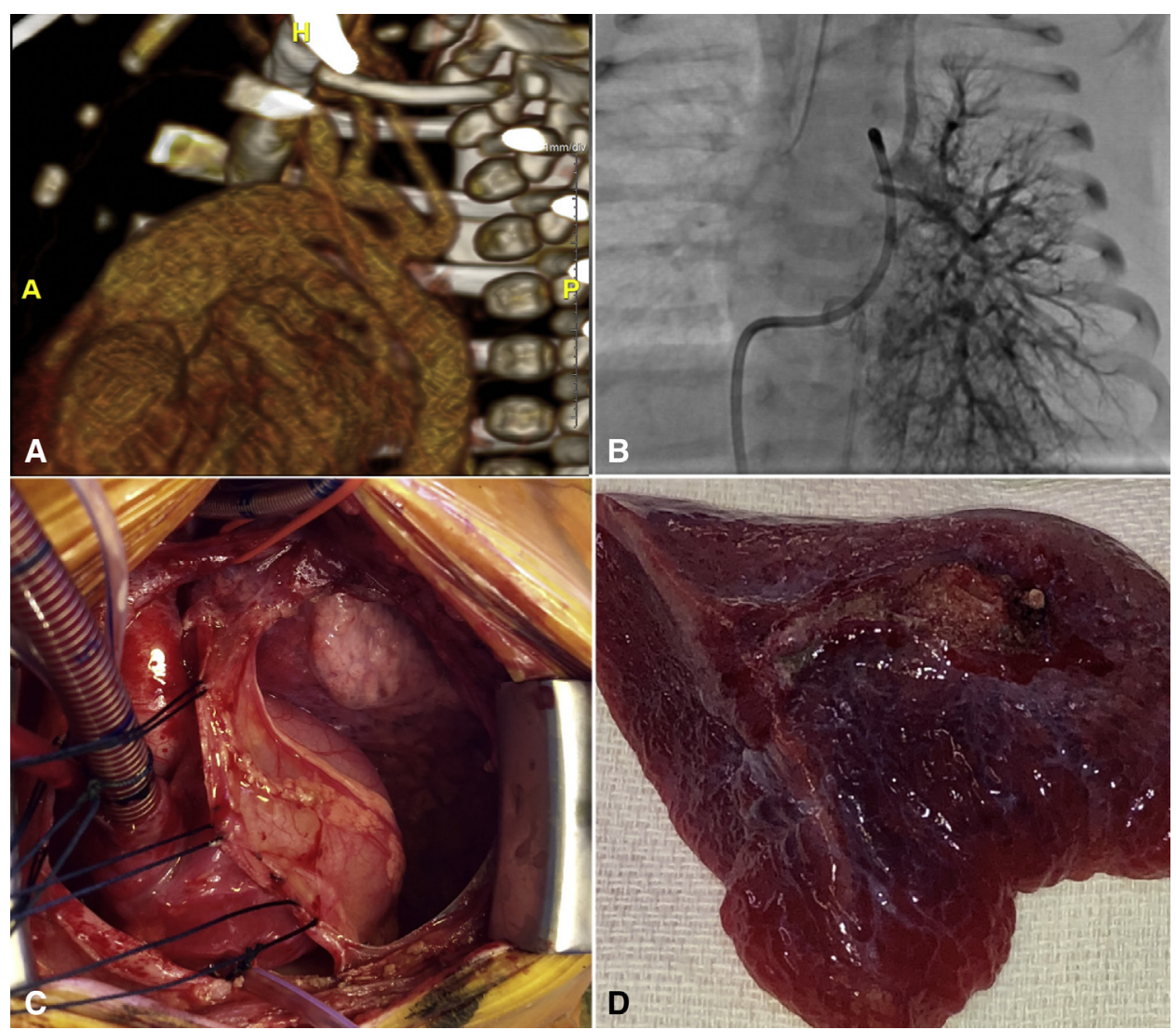

FIGURE 1. Unilateral pulmonary vein atresia with coarctation of the aorta is a very rare combination requiring a nuanced approach to surgical decision making. A, Chest X-ray shortly after birth demonstrating nearly complete opacification of the left lung. B, Pulmonary arteriogram demonstrating limited venous drainage to an atretic vertical vein and absence of a venous confluence, suggesting that pulmonary venous repair or augmentation would not provide a suitably durable result. C, Surgical exposure of the left hilum through a median sternotomy without evident pulmonary veins. A median sternotomy was selected because of the need for arch repair. D, After pneumonectomy, the medial aspect of the specimen demonstrated a boggy lung with no evident pulmonary veins.

endothelial cells lining these dilated vascular spaces expressed CD34, D2-40, and Prox-1. These features were deemed to be consistent with congenital pulmonary lymphangiectasia secondary to pulmonary vein atresia. The patient was last seen at follow-up 19 months postoperatively and was doing well.

\section{DISCUSSION}

Unilateral pulmonary vein atresia is a rare lesion, and diagnosis in the neonatal period is even rarer still. Given the poor prognosis for patients diagnosed with pulmonary vein atresia in infancy, surgical intervention is indicated. Approaches to the repair of this lesion include pulmonary venoplasty (either surgical or interventional), pneumonectomy, and lung transplantation.

Pulmonary venoplasty has been associated with a high rate of restenosis, ${ }^{1}$ and in the case presented here, the atretic veins were not amenable to augmentation owing to the atretic nature of the veins themselves and the associated vertical vein. Lung transplantation remains a possibility for patients with bilateral disease; however, given the risks associated with lung transplantation, including rejection, infection, and bronchiolitis obliterans, the decision to go to transplantation cannot be made lightly, and it should not be pursued in patients with unilateral disease. In addition, patients with associated cardiac lesions might not be able to wait for a donor allograft to become available. Pneumonectomy carries a significant risk for morbidities in children, including kyphoscoliosis, chest wall hypoplasia, bronchopleural fistula, postpneumonectomy pulmonary edema, and postpneumonectomy syndrome. However, given the otherwise substantial risk for mortality without intervention, the possible benefits far outweigh the potential risks.

An aggressive approach to the surgical management of neonatal patients with pulmonary vein atresia is possible. In patients with severe unilateral disease, pneumonectomy may be considered an alternative to either lung transplantation or venoplasty. In the setting of associated cardiac 
lesions, the threshold to perform additional lung resection should be low, given the otherwise poor prognosis.

\section{References}

1. Holt DB, Moller JH, Larson S, Johnson MC. Primary pulmonary vein stenosis. Am J Cardiol. 2007;99:568-72.
2. Tissot C, Corbelli R, Aggoun Y, Beghetti M, da Cruz E. Bronchoscopic diagnosis of asymptomatic unilateral pulmonary vein atresia in an infant. Pediatr Cardiol. 2008;29:976-9.

3. Song MK, Bae EJ, Jeong SI, Kang IS, Kim NK, Choi JY, et al. Clinical characteristics and prognostic factors of primary pulmonary vein stenosis or atresia in chil dren. Ann Thorac Surg. 2013;95:229-34.

4. Nakib A, Moller JH, Kanjuh VI, Edwards JE. Anomalies of the pulmonary veins. Am J Cardiol. 1967;20:77-90. 